\title{
Enhanced Visual Cortical Activation for Emotional Stimuli is Preserved in Patients with Unilateral Amygdala Resection
}

\author{
E. Kale Edmiston, ${ }^{1 \star}$ Maureen McHugo, ${ }^{1 \star}$ Mildred S. Dukic, ${ }^{2}$ Stephen D. Smith, ${ }^{3}$ Bassel Abou-Khalil, ${ }^{4}$ Erica Eggers, ${ }^{2}$ \\ and David H. Zald ${ }^{1,2}$ \\ ${ }^{1}$ Neuroscience Program and ${ }^{2}$ Department of Psychology, Vanderbilt University, Nashville, Tennessee 37240, ${ }^{3}$ Department of Psychology, University of \\ Winnipeg, Manitoba, R3B 2E9, and ${ }^{4}$ Department of Neurology, Vanderbilt University, Nashville, Tennessee 37240
}

\begin{abstract}
Emotionally arousing pictures induce increased activation of visual pathways relative to emotionally neutral images. A predominant model for the preferential processing and attention to emotional stimuli posits that the amygdala modulates sensory pathways through its projections to visual cortices. However, recent behavioral studies have found intact perceptual facilitation of emotional stimuli in individuals with amygdala damage. To determine the importance of the amygdala to modulations in visual processing, we used functional magnetic resonance imaging to examine visual cortical blood oxygenation level-dependent (BOLD) signal in response to emotionally salient and neutral images in a sample of human patients with unilateral medial temporal lobe resection that included the amygdala. Adults with right $(n=13)$ or left $(n=5)$ medial temporal lobe resections were compared with demographically matched healthy control participants $(n=16)$. In the control participants, both aversive and erotic images produced robust BOLD signal increases in bilateral primary and secondary visual cortices relative to neutral images. Similarly, all patients with amygdala resections showed enhanced visual cortical activations to erotic images both ipsilateral and contralateral to the lesion site. All but one of the amygdala resection patients showed similar enhancements to aversive stimuli and there were no significant group differences in visual cortex BOLD responses in patients compared with controls for either aversive or erotic images. Our results indicate that neither the right nor left amygdala is necessary for the heightened visual cortex BOLD responses observed during emotional stimulus presentation. These data challenge an amygdalo-centric model of emotional modulation and suggest that non-amygdalar processes contribute to the emotional modulation of sensory pathways.
\end{abstract}

\section{Introduction}

Emotionally salient stimuli capture greater attentional resources, can be perceived at lower stimulus thresholds, and are perceived more vividly than emotionally neutral stimuli (Phelps et al., 2006; Yiend, 2010; Todd et al., 2012). Functional magnetic resonance imaging (fMRI) studies consistently show enhanced ventral visual stream blood oxygen level-dependent (BOLD) signal during emotional stimulus presentations relative to neutral stimuli (Lang et al., 1998; Britton et al., 2006).

It has been postulated that the amygdala plays a crucial role in the enhanced processing of emotionally salient stimuli (Anderson and Phelps, 2001; Zald, 2003; Phan et al., 2004; Reinders et al., 2005; Fitzgerald et al., 2006; Peck et al., 2013). In nonhuman primates, anatomical tracing studies have found topographically

Received Jan. 25, 2013; revised May 10, 2013; accepted May 27, 2013.

Author contributions: E.K.E., M.M., and D.H.Z. designed research; E.K.E. and M.M. performed research; E.K.E., M.M., M.S.D., S.D.S., B.A.-K., and E.E. analyzed data; E.K.E., M.M., and D.H.Z. wrote the paper.

This work was supported by grants from the National Institute of Mental Health (R01 MH074567) to D.H.Z. and from the Center for Advancing Translational Sciences (UL1 TR000445) to the Vanderbilt Institute of Clinical and Translational Research and a fellowship from The Clinical Neuroscience Scholars Program at Vanderbilt University to E.K.E.

The authors declare no competing financial interests.

${ }^{*}$ E.K.E. and M.M. contributed equally to this work.

Correspondence should be addressed to Dr. David H. Zald, Department of Psychology, PMB 407817111 21st Avenue, South Nashville TN 37240-7817. E-mail: david.zald@vanderbilt.edu.

DOI:10.1523/JNEUROSCI.0401-13.2013

Copyright $\odot 2013$ the authors $\quad 0270-6474 / 13 / 3311023-09 \$ 15.00 / 0$ organized, ipsilateral projections from the basolateral amygdala to ventral visual stream areas (Amaral et al., 2003). These projections have been proposed to represent a key mechanism through which the amygdala can facilitate perception of, and attention to, emotionally salient stimuli based on rapid stimulus evaluation (for review, see Tamietto and de Gelder, 2010). In support of this model, neuroimaging studies using functional connectivity analyses demonstrate coupling between amygdalar and visual stream BOLD signal in human subjects viewing emotional stimuli (Das et al., 2005; Wendt et al., 2011; although the causal feedforward vs feedback direction of the associations cannot be determined in these studies). Perhaps the strongest support for the idea that the amygdala modulates visual cortex processing comes from Vuilleumier et al. (2004), who reported that epileptic patients with medial temporal lobe sclerosis involving the amygdala showed reduced ipsilateral fusiform and visual cortex activations in response to fear faces. Benuzzi et al. (2004) also demonstrated reduced visual cortex activations in response to fearful faces in patients with early onset epilepsy and right (but not left) medial temporal lobe sclerosis.

However, recent behavioral studies of patients with either bilateral amygdala lesions (Tsuchiya et al., 2009; Bach et al., 2011) or unilateral amygdala resection (Piech et al., 2010) have reported intact emotional facilitation of visual search and target detection tasks. Reviewing the extant literature, Pessoa and Adolphs (2010) call into question the relative importance of the amygdala in the 
Table 1. Patient demographic data

\begin{tabular}{|c|c|c|c|c|c|c|}
\hline Group & $\begin{array}{l}\text { Surgery type } \\
\text { SAH (TL) }\end{array}$ & $\begin{array}{l}\text { Male } \\
\text { (total) }\end{array}$ & $\begin{array}{l}\text { Mean years of } \\
\text { age } \pm S D\end{array}$ & $\begin{array}{l}\text { Mean years of } \\
\text { education } \pm S D\end{array}$ & $\begin{array}{l}\text { Mean years from } \\
\text { surgery } \pm S D\end{array}$ & $\begin{array}{l}\text { Mean age of } \\
\text { onset } \pm S D\end{array}$ \\
\hline \multicolumn{7}{|l|}{ Patients } \\
\hline Left resection & $1(4)$ & $3(5)$ & $36.40 \pm 9.30$ & $13.60 \pm 1.67$ & $8.32 \pm 6.24$ & $7.10 \pm 5.06$ \\
\hline Controls & $\mathrm{N} / \mathrm{A}$ & $6(16)$ & $39.38 \pm 7.59$ & $14.13 \pm 2.03$ & $\mathrm{~N} / \mathrm{A}$ & $\mathrm{N} / \mathrm{A}$ \\
\hline
\end{tabular}

SAH, selective amygdalohippocampectomy; $\mathrm{TL}$, temporal lobectomy. Mean age of onset refers to age of seizure onset.

emotional guidance of visual processing, suggesting other mechanisms may be as or more important than an amygdala-mediated process, and that a network of associated regions may be implicated in the emotional modulation of visual processing.

In the present study we assessed visual cortex BOLD responses to emotional scenes in a sample of patients with unilateral amygdala lesions. We focused on emotionally salient scenes because of their ability to produce a broader engagement of visual regions than faces (Sabatinelli et al., 2011). If the amygdala is necessary for emotional modulation of sensory areas, patients with resection of the amygdala should fail to show an enhanced response to emotional stimuli in visual areas ipsilateral to the amygdala lesion. In contrast, if enhancement remains following amygdala lesions, it would support Pessoa and Adolphs' (2010) argument that the amygdala plays a less essential or general role than previously thought.

\section{Materials and Methods}

Participants. Participants were adults with pharmacologically intractable medial temporal lobe epilepsy who had previously undergone unilateral surgical resection involving the amygdala and healthy adults matched on demographic factors to the resection patients. Eighteen patients were recruited from the Vanderbilt University Medical Center Epilepsy Surgery Program (for demographic information, see Table 1). All participants had an extensive history of intractable epilepsy before surgery, with an average onset of seizure $\sim 8$ years of age, but surgery not occurring until adulthood. Resections consisted of unilateral anterior temporal lobectomy (left $=4$; right $=9$ ) or unilateral selective amygdalohippocampectomy (left $=1$; right $=4$, see Fig. 1 ). The length of time since surgery was variable with patients receiving selective resections having a shorter time since surgery ( mean $=2.66$ years) than those receiving lobectomies (mean $=10.87$ years) due to historical trends in surgical procedures. Three participants were unmedicated at the time of scanning. The remaining participants were prescribed levetiracetam $(n=5)$, carbamazepine $(n=3)$, trileptal $(n=4)$, lamotrigine $(n=6)$, mirapex $(n=1)$, ziprasidone $(n=1)$, Lyrica $(n=2)$, topiramate $(n=2)$, gabapentin $(n=$ 2 ) or selective serotonin reuptake inhibitors/serotonin-norepinephrine reuptake inhibitor $(n=4)$. The healthy control group $(n=16)$ was recruited from the Nashville community using on-line advertisements. Controls were matched for age, sex, and education with the patient group and had no self-reported major Axis I psychopathology. Participants with generalized cognitive impairment (IQ $<80$ ) were excluded, as were resection patients with neurological conditions other than epilepsy or lesions outside of the temporal lobe. All participants gave written informed consent to participate in the study. This study was approved by the Vanderbilt University Institutional Review Board.

fMRI task. Participants completed two 4 min runs of a block design passive viewing task. Subjects viewed alternating blocks of aversive, erotic, neutral, and scrambled images taken from the International Affective Picture System (IAPS; Lang et al., 2008). The erotic condition was supplemented with images of couples in erotic poses downloaded from the Internet to ensure they were of high arousal and did not feel "dated." The scrambled condition was created in Adobe Photoshop by applying a filter to the aversive images that retained the color and luminosity but blurred the picture so that it lacked recognizable features. Each run was composed of 16 blocks with 4 blocks of each of the four conditions. Images from each condition (aversive, erotic, neutral, or scrambled) were presented in $16 \mathrm{~s}$ blocks, with four images in each block at $1024 \times$ 768 pixels, subtending $34.2 \times 32.6$ degrees of visual angle. Images were projected on to a mirror that was attached to the scanner head coil, $\sim 33$ $\mathrm{cm}$ from the subject's eyes. Image duration was $4 \mathrm{~s}$ and each image was presented only once. All images were matched for luminance and complexity. Aversive and erotic images were matched for arousal ratings provided by IAPS or pilot ratings for supplemented erotic images.

$f M R I$ data. Structural and functional images were collected on one of two identical 3 T Phillips Intera Achieva Scanners (Phillips Healthcare) using an 8-channel head coil. Because two patients and two control participants were scanned on an alternate scanner, the scanner was included as a covariate of no interest for all analyses to control for potential confounds. The scan session included high-resolution T1-weighted anatomical images with the following parameters: $256 \mathrm{~mm}$ FOV, 170 slices, 1 mm slice thickness, no gap, as well as echoplanar images TR $=2000 \mathrm{~ms}$, $\mathrm{TE}=35 \mathrm{~ms}$, 90 degree flip angle, FOV $=240 \mathrm{~mm}, 128 \times 128 \mathrm{~mm}$ resolution.

Data were preprocessed using SPM8 (http://www.fil.ion.ucl.ac. $\mathrm{uk} / \mathrm{spm}$ ) and MATLAB (Version 7.1; MathWorks). Data were slice-time corrected, motion-corrected (aligned to the mean image across runs), and coregistered with the structural image. Images were normalized to the Montreal Neurological Institute (MNI) T1 template using unified segmentation and normalization (Ashburner et al., 2005; Crinion et al., 2007) and resampled to $2 \mathrm{~mm}^{3}$ voxels. Data were smoothed using a $5 \mathrm{~mm}$ full-width at half-maximum Gaussian kernel. All participants included for analysis had translation $<2 \mathrm{~mm}$ and rotation $<2$ degrees.

Whole-brain statistical analysis was performed using the general linear model in SPM8. Separate regressors were constructed modeling each of the three stimulus types (erotic, aversive, neutral) against the scrambled image condition baseline. We then created separate contrast images comparing the aversive versus neutral, erotic versus neutral, and neutral versus scrambled conditions, averaging across runs. The neutral versus scrambled contrast was included to ensure that there were no overall differences in visual processing between the groups. These first (participant)-level contrast images were then used for second (group)level whole-brain analysis. Within-group activation was examined by separate analyses of each contrast (aversive $>$ neutral, erotic $>$ neutral, neutral $>$ scrambled) in each group (right-sided resection, left-sided resection, healthy controls), using one sample $t$ tests.

We performed a between-group analysis comparing the erotic $>$ neutral and aversive $>$ neutral contrast maps of left and right amygdala resection patients. To increase statistical power to detect alterations in responses relative to the control group, we created a combined lesion group. To accomplish this, the contrast maps for the five left-sided resection patients were flipped along the $x$-axis before being combined with the right hemisphere lesion patients. In all results involving the combined resection group, findings in the right hemisphere reflect ipsilateral effects. Between-group analyses were then performed between the combined lesion group and the healthy controls. Results were corrected for multiple comparisons using topological false discovery rate (FDR) of $q<0.05$ (Chumbley and Friston, 2009).

To explore potential effects of remaining amygdala volume on BOLD signal in the combined patient sample, we performed a robust regression analysis using the Robust Toolbox for SPM (Wager et al., 2005) as an additional post hoc analysis (topological FDR of $q<0.05$ ). Percentage signal was extracted from three $10 \mathrm{~mm}$ diameter spherical regions of interest in the primary visual cortex, right fusiform gyrus, and left visual association cortex, with the center of the regions of interest (ROIs) de- 


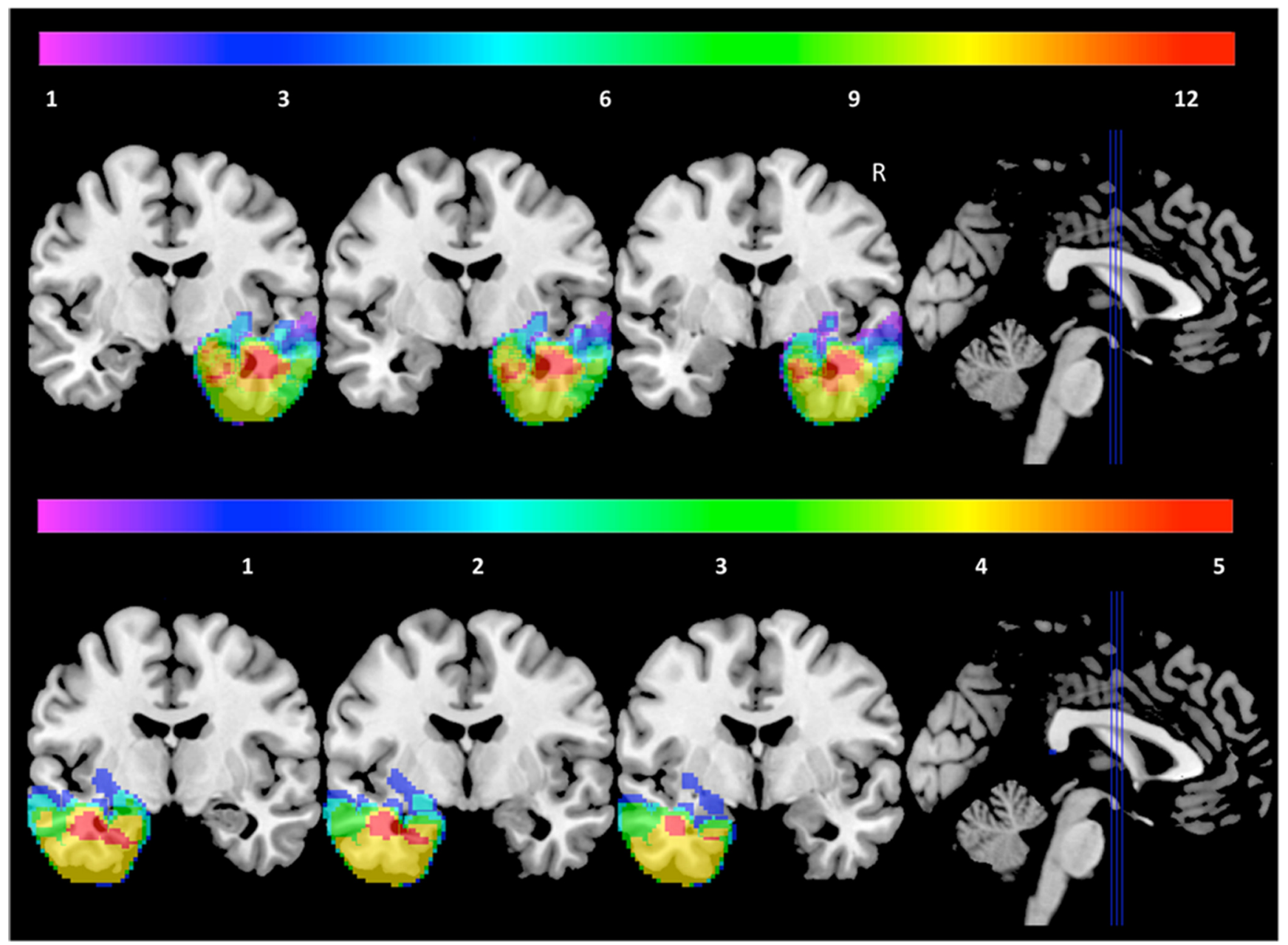

Figure 1. Extent of overlap of resected area in right resection patients (top) and left resection patients (bottom). Color bar and numbers represent number of patients.

termined by the result arising in the primary contrasts. Amygdala volume remaining on the lesion side was calculated as a percentage of the contralateral amygdala for each subject. Volumes were generated via hand tracings (M.M.). Amygdala volumes were manually traced on the normalized structural images of each participant using FSLView (http://www.fmrib.ox.ac.uk/fsl/fslview/index.html). Amygdala boundaries were determined based on criteria modified from Pruessner et al. (2000) and Honeycutt et al. (1998) as previously described by our group (Piech et al., 2011).

\section{Results}

Figure 1 displays the lesion overlap in the patients with amygdala damage. Volumetric analyses revealed that an average of 88.14 and $83.59 \%$ of the normal volume of the amygdala was missing in the right and left resection groups respectively, with nine patients showing complete unilateral amygdala loss.

We first examined activation for the aversive $>$ neutral and erotic $>$ neutral contrasts within the healthy control group (all results topological FDR corrected, $q<0.05$ ). Consistent with their strong emotional features, aversive images elicited greater bilateral amygdala activation than neutral images (Fig. 2). As expected, the aversive $>$ neutral contrast revealed significant BOLD signal increases in bilateral primary and secondary visual cortices, including lateral occipital regions, and the fusiform gyrus (see Fig. 3, Tables 2, 3). Additional increased activations were present in left amygdala and hippocampus, left inferior parietal cortex, left somatosensory cortex, midbrain, posterior thal- amus, bilateral caudate-putamen, right postcentral gyrus, and left inferior frontal gyrus (Tables 4,5 ). The erotic $>$ neutral contrast also showed increased BOLD signal in the bilateral primary and secondary visual cortices (Fig. 3). Additional activations emerged in multiple brain regions including the precuneus and a large cluster extending from the midbrain through the hippocampus and bilateral amygdala (Fig. 2), left temporal pole, bilateral caudate-putamen, left premotor cortex, right postcentral gyrus, left middle frontal gyrus, right inferior frontal gyrus, and the anterior cingulate gyrus (Tables 2-5).

Between-group analyses did not show significant differences between the left and right resection groups across both the aversive $>$ neutral and erotic $>$ neutral contrasts even at a liberal uncorrected threshold of $p<0.001, k=10$. Therefore, the left patient group contrast maps were flipped along the $x$-axis and combined with the right patient group to increase power to detect group effects for all further analyses. In reporting results from the combined group, ipsilateral effects are expressed in the right hemisphere, and contralateral effects occur in the left hemisphere. Analysis of the combined $(n=18)$ resection patient group demonstrated that BOLD signal was significantly increased for aversive compared with neutral images in regions including bilateral primary and secondary visual cortices (Fig. 3, Tables 2,3). Greater activation for aversive images was also found in the contralateral inferior parietal cortex, posterior thalamus, contralateral insula, and bilateral inferior frontal gyri (Fig. 3, Ta- 
bles 4, 5). For the erotic $>$ neutral contrast, the combined patient group showed significant BOLD signal increase in the bilateral primary and secondary visual cortices, brainstem, midbrain, ipsilateral posterior thalamus, a large cluster extending from the insular cortex to the amygdala and hippocampus, ipsilateral frontal eye fields, contralateral middle frontal gyrus, bilateral inferior frontal gyrus, and the anterior cingulate gyrus (Fig. 3, Tables 2-5).

To determine whether there were any differences in visual cortex activations in the hemisphere ipsilateral versus contralateral to the amygdala lesion, we extracted the percentage BOLD signal change for each subject in regions of interest centered on three peak areas of visual cortical activation, including a primary visual region, lateral visual association cortex, and the fusiform gyrus, both ipsilateral and contralateral to the amygdala lesion. Paired $t$ tests did not demonstrate any differences between the ipsilateral and contralateral BOLD responses for either the aversive relative to neutral or erotic relative to neutral conditions, although there was a significant trend for greater contralateral than ipsilateral fusiform gyrus activation in the erotic-neutral contrast (Table 6).

Between-group analysis of the amygdala resection patients versus healthy controls did not show any significant increases or decreases in activation in any brain region in either the aversive $>$ neutral, erotic $>$ neutral, or neutral $>$ scrambled contrasts. This finding held regardless of whether each amygdala resection group was compared separately to healthy controls or if they were combined into a single group. At a liberal threshold for statistical significance $(p<0.001, k=10)$, one small cluster in the right fusiform gyrus (ipsilateral to the lesion, cluster size $=28$ voxels, maxima at $x=34, y=-34, z=-22$ ) displayed increased activation in the aversive $>$ neutral contrast in the controls relative to the combined resection patients (Fig. 4).

Although there were no statistically significant group differences between the patient and control groups, visual inspection of Figure 3 , and the cluster sizes reported in Tables 2 and 3, suggest there was a greater extent of visual cortical activation during the aversive versus neutral contrast for the controls relative to the patients. Examination of individual subject data revealed that a single patient heavily drove this reduction in size. This participant had a complete amygdala lesion. However, the other eight patients with complete unilateral resections of the amygdala all showed normal levels of visual cortical activation to aversive stimuli (Fig. 5), indicating that lesion size (or location within the amygdala) did not explain this one subject's lack of a strong response to aversive stimuli. This participant was also not unique in terms of demographics or medications. Critically, even though this patient showed no enhancement of processing of aversive stimuli, the patient showed enhanced activation of visual regions to erotica, indicating that there was not a generalized deficit in the ability of emotionally salient stimuli to modulate visual regions.

To explore whether lesion volume contributes to differences in the extent to which emotionally salient stimuli receive preferential processing, we performed a robust regression analysis with percentage amygdala loss on the resected side serving as the independent variable. For the aversive versus neutral contrast, this analysis revealed a significant positive correlation between the extent of amygdala resection and BOLD signal in a small cluster in the left (i.e., contralateral to the lesion) fusiform gyrus (Table 7). Thus rather than reducing activations, larger resections were associated with increased BOLD signal $\left(r^{2}=0.45\right)$ in this region. Greater amygdala volume loss was also associated with increased activation of the ipsilateral precuneus, ipsilateral temporal parietal junction, ipsilateral superior parietal cortex, and contralateral ventral frontopolar cortex. In contrast, there was a significant negative correlation between BOLD signal in the ipsilateral posterior thalamus and extent of amygdala resection. For the erotic $>$ neutral contrast, there was a significant positive correlation between extent of resection and BOLD signal in the contralateral visual association cortex $\left(r^{2}=0.11\right)$ and contralateral inferior parietal cortex. This indicates that patients with larger lesions showed an enhanced activation contralateral to the lesion. The only area in which lesion extent was associated with reduced activation during viewing of erotic stimuli was in the contralateral temporal parietal junction.

A limitation of most clinical neuropsychology studies is that the studies are conducted long after the start of pathology, which means that neural plasticity may allow for compensatory changes. This issue takes on added importance given that Benuzzi et al. (2004) have suggested that age of seizure onset may influence whether there is preservation of the emotional facilitation in processing faces, based on a small study of patients with medial temporal sclerosis. We therefore performed additional regression 


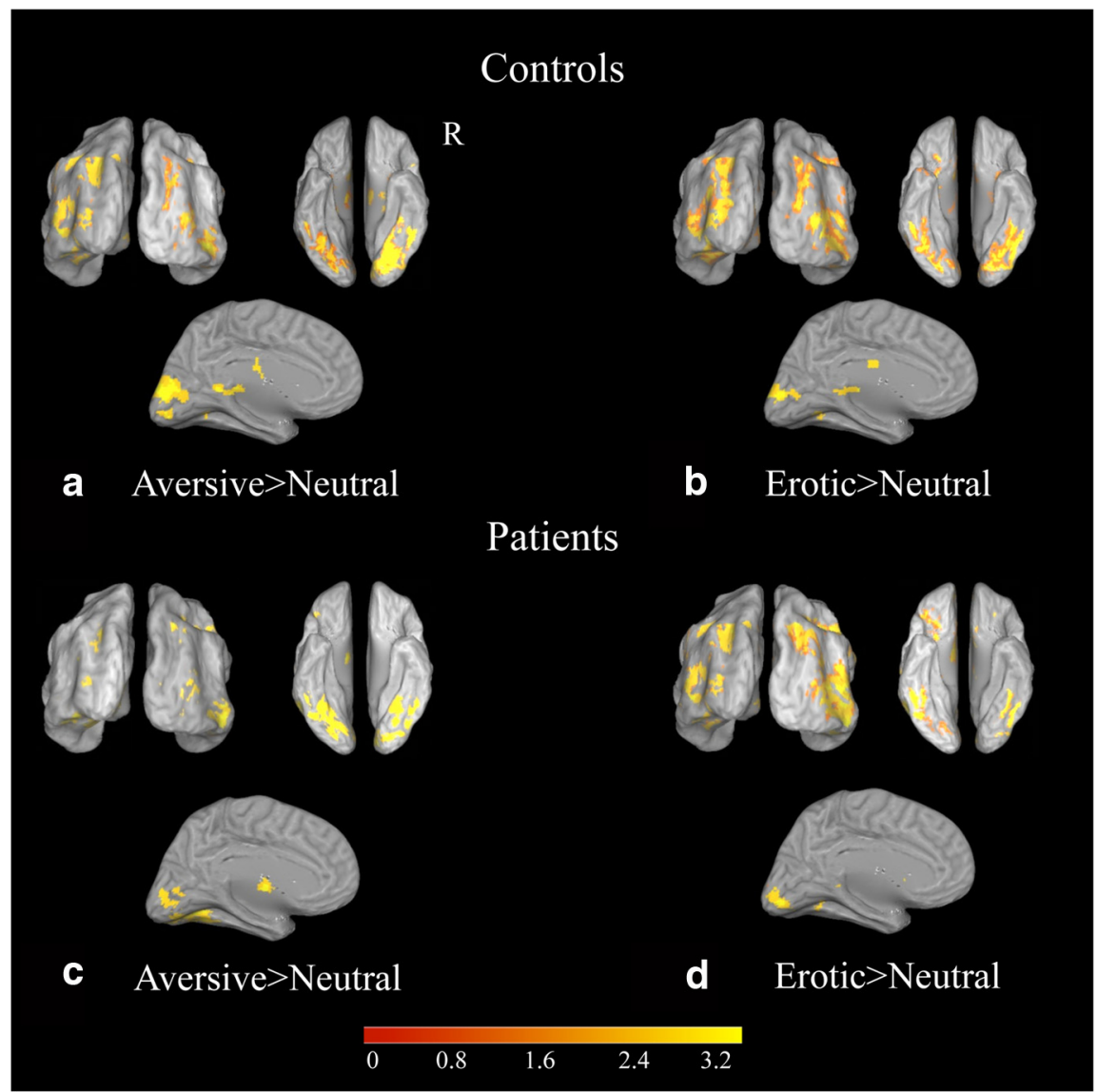

Figure 3. Inflated brains in posterior, ventral, and medial views demonstrate areas of increased BOLD signal for emotionally salient conditions relative to neutral in visual cortex in (a) controls, aversive $>$ neutral; $(\boldsymbol{b})$ controls, erotic $>$ neutral; $(\boldsymbol{c})$ combined left and right patients, aversive $>$ neutral; and $(\boldsymbol{d})$ combined left and right patients erotic $>$ neutral. All results topological FDR corrected, $q<0.05$.

analyses with age of seizure onset as the independent variable. We similarly performed these analyses with time since surgery as the independent variable. There were no significant correlations (all $p>0.10$ ) between either of these measures and BOLD signal in spherical ROIs in the bilateral fusiform gyrus or in primary visual cortex for both the aversive $>$ neutral or the erotic $>$ neutral contrasts. Thus, while the large length of time since seizure onset and/or surgical resection could allow for plastic compensatory changes in emotional facilitation, there was no evidence that the preservation of visual responses to emotional stimuli tracked with either the age at which seizures began or the amount of time since surgery.

\section{Discussion}

In this study we directly tested whether the amygdala plays an essential role in the enhancement of visual cortical responses to emotional stimuli. As expected, in the control population, we found enhanced BOLD signal in response to emotionally salient stimuli in primary and secondary visual cortices. Strikingly, we observed similar activation patterns in the amygdala resection sample, with no significant differences emerging between groups, even in visual cortex ipsilateral to the lesion. Given its role in emotional processing and its projections to visual regions, the amygdala has been assumed to be the key region directing enhancement of visual processing to emotional stimuli (Morris et al., 1998). However, our findings suggest that the modulation of BOLD activity in visual cortices does not depend on the amygdala. This finding is in line with the recent proposal put forth by Pessoa and Adolphs (2010), which argues that processing of emotional stimuli occurs in multiple parallel waves, and is facilitated by a network of associated structures, including the orbitofrontal cortex, insula, and pulvinar nucleus of the thalamus. As such, multiple regions capable of modulating visual pathways may evaluate visual information to ensure that perceptual processing is directed to the most relevant stimuli. In this model, the amygdala may still influence a number of aspects of visual processing, but is not essential to the enhancement of visual cortex activation.

Our overall findings contrast those of Vuilleumier et al. (2004), who concluded that the amygdala plays a necessary role in emotional modulation of visual processing based on observed reductions in responses to fear faces ipsilateral to amygdalar sclerosis. We note three critical differences between our study and the Vuilleumier study. Consideration of these differences may prove helpful in specifying the nature and limitations of amygdalar influences on visual processing. First, the use of complex emotional scenes instead of faces in our study likely contributes to differences given that complex scenes are more emotionally arousing than emotional faces and induce more widespread visual cortex activation (Britton et al., 2006; Sabatinelli et al., 2011). Consistent with data demonstrating functional connectivity between the amygdala and fusiform gyrus during viewing of emotional faces (Fairhall and Ishai, 2007), the Vuilleumier et al. (2004) findings appear strongest in the fusiform gyrus; it may be that the amygdala exerts greater control over fusiform gyrus during emotional processing than other visual areas. Indeed, a recent fMRI study in monkeys demonstrated that lesions of the central nucleus of the amygdala eliminate valence effects in inferior temporal regions that are sensitive to faces (Hadj-Bouziane et al., 2012). Interestingly, we observed a small cluster in the right fusiform gyrus (ipsilateral to resection) in our group comparison for the aversive-neutral contrast with a liberal statistical threshold. This finding, although nonsignificant at more rigorous thresholds, could reflect a highly localized decrease in the ipsilateral modulation of fusiform processing in patients with amygdala lesions.

Another critical difference is that the Vuilleumier study used an attentional manipulation, with faces and houses appearing at task-relevant or -irrelevant positions. It may be that under situations with top-down attentional demands, the amygdala facilitates perception of emotional stimuli, but is unnecessary in nondemanding free viewing conditions during which other mechanisms may contribute to the emotional modulation of sensory regions. We cannot rule out the possibility that patients voluntarily devoted greater top-down selective or spatial attentional resources to the emotional scenes (which would result in enhanced visual cortex activity) given that we did not explicitly control attention. However, we did not find increased activation 
Table 2. Cluster maxima and submaxima for visual cortical regions of statistically significant increased BOLD signal in emotionally salient conditions relative to neutral for controls and patients, aversive $>$ neutral

\begin{tabular}{|c|c|c|c|c|c|c|c|c|c|c|c|c|c|}
\hline \multicolumn{7}{|l|}{ Control group } & \multicolumn{7}{|l|}{ Patient group } \\
\hline \multirow{2}{*}{$\frac{\text { Aversive }>\text { neutral }}{\text { Brain regions (hemisphere) }}$} & \multirow[b]{2}{*}{ Cluster size } & \multirow[b]{2}{*}{$p$ value $^{*}$} & \multicolumn{4}{|c|}{ Peak voxel } & \multirow{2}{*}{$\frac{\text { Aversive }>\text { neutral }}{\text { Brain regions (hemisphere) }}$} & \multirow[b]{2}{*}{ Cluster size } & \multirow[b]{2}{*}{$p$ value } & \multicolumn{4}{|c|}{ Peak voxel } \\
\hline & & & tscore & $x$ & $y$ & $z$ & & & & tscore & $x$ & $y$ & $z$ \\
\hline \multirow[t]{3}{*}{ Occipital cortex (bilateral) } & 6396 & $<0.0001$ & 7.96 & -40 & -56 & -8 & Occipital cortex (bilateral) & 3521 & $<0.0001$ & 6.71 & -30 & -54 & -12 \\
\hline & & & & 32 & -70 & 26 & & & & & -46 & -50 & -18 \\
\hline & & & & 6 & -76 & -12 & & & & & 30 & -72 & -16 \\
\hline \multirow[t]{6}{*}{ Fusiform gyrus (left) } & 917 & $<0.0001$ & 6.22 & -36 & -84 & 8 & Occipital cortex (contralateral) & 161 & $<0.0001$ & 4.87 & -32 & -84 & 16 \\
\hline & & & & -28 & -74 & 26 & & & & & -30 & -90 & 4 \\
\hline & & & & -26 & -78 & 36 & & & & & -40 & -82 & 4 \\
\hline & & & & & & & Occipital cortex (ipsilateral) & 204 & $<0.0001$ & 4.86 & 24 & -64 & 38 \\
\hline & & & & & & & & & & & 24 & -72 & 36 \\
\hline & & & & & & & & & & & 26 & -66 & 22 \\
\hline
\end{tabular}

*Topological FDR corrected value. For patient contrasts, laterality is indicated in reference to lesion site.

Table 3. Cluster maxima and submaxima for visual cortical regions of statistically significant increased BOLD signal in emotionally salient conditions relative to neutral for controls and patients, erotic $>$ neutral

\begin{tabular}{|c|c|c|c|c|c|c|c|c|c|c|c|c|c|}
\hline \multicolumn{7}{|l|}{ Control group } & \multicolumn{7}{|l|}{ Patient group } \\
\hline \multirow{2}{*}{$\begin{array}{l}\text { Erotic }>\text { neutral } \\
\text { Brain regions (hemisphere) }\end{array}$} & \multirow[b]{2}{*}{ Cluster size } & \multirow[b]{2}{*}{$p$ value } & \multicolumn{4}{|c|}{ Peak voxel } & \multirow{2}{*}{$\begin{array}{l}\text { Erotic }>\text { neutral } \\
\text { Brain regions (hemisphere) }\end{array}$} & \multirow[b]{2}{*}{ Cluster Size } & \multirow[b]{2}{*}{$p$ value } & \multicolumn{4}{|c|}{ Peak voxel } \\
\hline & & & $t$ score & $x$ & $y$ & $z$ & & & & tscore & $x$ & $y$ & $Z$ \\
\hline \multirow[t]{6}{*}{ Occipital cortex (bilateral) } & 10265 & $<0.0001$ & 9.19 & -34 & -86 & -4 & Occipital cortex (bilateral) & 10502 & $<0.0001$ & 8.17 & -46 & -50 & -18 \\
\hline & & & & 32 & -70 & 28 & & & & & -40 & -40 & 40 \\
\hline & & & & -48 & -66 & 6 & & & & & -50 & -62 & -6 \\
\hline & & & & & & & Extrastriate cortex (ipsilateral) & 231 & $<0.0001$ & 4.80 & 10 & -80 & 2 \\
\hline & & & & & & & & & & & -2 & -84 & -8 \\
\hline & & & & & & & & & & & -8 & -78 & -2 \\
\hline
\end{tabular}

For patient contrasts, laterality is indicated in reference to lesion site.

Table 4. Cluster maxima for all other regions of statistically significant increased BOLD signal in emotionally salient conditions relative to neutral for controls and patients, aversive $>$ neutral

\begin{tabular}{|c|c|c|c|c|c|c|c|c|c|c|c|c|c|}
\hline \multicolumn{7}{|l|}{ Control group } & \multicolumn{7}{|l|}{ Patient group } \\
\hline \multirow{2}{*}{$\frac{\text { Aversive }>\text { neutral }}{\text { Brain regions (hemisphere) }^{*}}$} & \multirow{2}{*}{$\begin{array}{l}\text { Cluster } \\
\text { size }\end{array}$} & \multirow[b]{2}{*}{$p$ value $^{* *}$} & \multicolumn{4}{|c|}{ Peak voxel } & \multirow{2}{*}{$\frac{\text { Aversive }>\text { neutral }}{\text { Brain regions (hemisphere) }}$} & \multirow{2}{*}{$\begin{array}{l}\text { Cluster } \\
\text { size }\end{array}$} & \multirow[b]{2}{*}{$p$ value } & \multicolumn{4}{|c|}{ Peak voxel } \\
\hline & & & tscore & $x$ & $y$ & $z$ & & & & $t$ score & $x$ & $y$ & $z$ \\
\hline Midbrain (bilateral) & 763 & $<0.0001$ & 7.30 & -6 & -32 & -14 & Posterior thalamus (contralateral) & 96 & 0.005 & 6.27 & -8 & -8 & 2 \\
\hline Amygdala (left) & 104 & 0.003 & 6.50 & -20 & -4 & -16 & Inferior frontal gyrus (contralateral) & 355 & $<0.0001$ & 5.61 & -48 & 12 & 24 \\
\hline Posterior thalamus (left) & 193 & $<0.0001$ & 6.32 & -10 & -16 & 4 & Inferior parietal cortex (contralateral) & 445 & $<0.0001$ & 5.43 & -26 & -60 & 42 \\
\hline Hippocampus (left) & 88 & 0.005 & 6.04 & -24 & -32 & -6 & Inferior frontal gyrus (ipsilateral) & 139 & 0.001 & 5.31 & 46 & 12 & 28 \\
\hline Inferior frontal gyrus (right) & 428 & $<0.0001$ & 6.02 & 56 & 14 & 30 & Inferior frontal gyrus (contralateral) & 64 & 0.027 & 4.84 & -44 & 18 & -10 \\
\hline Posterior thalamus (right) & 154 & $<0.0001$ & 5.82 & 10 & -18 & 12 & Insula (contralateral) & 54 & 0.043 & 4.54 & -38 & -2 & -6 \\
\hline Midbrain (left) & 87 & 0.005 & 5.43 & -2 & -4 & -16 & Inferior frontal gyrus (contralateral) & 100 & 0.005 & 4.45 & -46 & 30 & 18 \\
\hline Putamen (right) & 83 & 0.006 & 5.41 & 32 & -10 & -8 & & & & & & & \\
\hline Caudate-putamen (left) & 112 & 0.002 & 5.04 & -8 & 4 & -2 & & & & & & & \\
\hline Somatosensory cortex (left) & 263 & $<0.0001$ & 5.00 & -56 & -30 & 30 & & & & & & & \\
\hline Inferior frontal gyrus (left) & 128 & 0.001 & 4.87 & -44 & 38 & 6 & & & & & & & \\
\hline Caudate-putamen (left) & 126 & 0.001 & 4.84 & -16 & 0 & 12 & & & & & & & \\
\hline Postcentral gyrus (right) & 66 & 0.015 & 4.59 & 50 & -26 & 42 & & & & & & & \\
\hline Caudate-putamen (right) & 205 & $<0.0001$ & 4.54 & 10 & 2 & 12 & & & & & & & \\
\hline Inferior frontal gyrus (left) & 51 & 0.033 & 4.44 & -34 & 10 & 24 & & & & & & & \\
\hline Inferior parietal cortex (left) & 59 & 0.021 & 4.41 & -38 & -42 & 44 & & & & & & & \\
\hline
\end{tabular}

For patient contrasts, laterality is indicated in reference to lesion site. *For brevity, submaxima are not reported but are available upon request. ${ }^{* *}$ Topological FDR corrected value.

in areas typically associated with top-down attention (e.g., frontal eye fields or intraparietal sulcus) in patients relative to controls in either the emotional or neutral conditions, suggesting top-down attentional affects are not a primary explanation for our findings.

Furthermore, in this study we used a block design, whereas the Vuilleumier study used an event-related design. It is possible that the amygdala plays a role in a rapid attentional manipulation (observable at a single trial level), but that more sustained modulations through a block of trials can be modulated by other brain regions. Electrophysiological studies of patients with implanted electrodes suggest that the amygdala possesses both a rapid transient and a slower sustained response to emotional stimuli (Krolak-Salmon et al., 2004), with responses in cortical areas including orbitofrontal, inferior temporal, and visual areas following a slower course. Based on data from a rapid sampling fMRI study, Sabatinelli et al. (2009) suggest that there may be two peaks of neural activation in visual pathways following presentation of emotional stimuli. They propose that the first peak is 
Table 5. Cluster maxima for all other regions of statistically significant increased BOLD signal in emotionally salient conditions relative to neutral for controls and patients, erotic > neutral

\begin{tabular}{|c|c|c|c|c|c|c|c|c|c|c|c|c|c|}
\hline \multicolumn{7}{|l|}{ Control group } & \multicolumn{7}{|l|}{ Patient group } \\
\hline \multirow{2}{*}{$\begin{array}{l}\text { Erotic }>\text { neutral } \\
\text { Brain regions (hemisphere) }\end{array}$} & \multirow[b]{2}{*}{ Cluster size } & \multirow[b]{2}{*}{$p$ value } & \multicolumn{4}{|c|}{ Peak voxel } & \multirow{2}{*}{$\frac{\text { Erotic }>\text { neutral }}{\text { Brain regions (hemisphere) }}$} & \multirow{2}{*}{$\begin{array}{l}\text { Cluster } \\
\text { size }\end{array}$} & \multirow[b]{2}{*}{$p$ value } & \multicolumn{4}{|c|}{ Peak voxel } \\
\hline & & & tscore & $x$ & $y$ & $z$ & & & & tscore & $x$ & $y$ & $z$ \\
\hline Amygdala-hippocampus-midbrain (left) & 1079 & $<0.0001$ & 9.06 & -22 & -4 & -14 & $\begin{array}{l}\text { Amygdalo-hippocampus-insular cortex } \\
\text { (contralateral) }\end{array}$ & 2250 & $<0.0001$ & 7.26 & -32 & -16 & -4 \\
\hline Caudate-putamen (bilateral) & 968 & $<0.0001$ & 7.16 & 18 & 12 & 4 & Inferior frontal gyrus (contralateral) & 1171 & $<0.0001$ & 7.15 & -46 & 10 & 24 \\
\hline Orbitofrontal (left) & 72 & 0.011 & 6.68 & -28 & 14 & -18 & Anterior cingulate cortex (bilateral) & 1315 & $<0.0001$ & 6.38 & 0 & 46 & 18 \\
\hline Anterior cingulate cortex (left) & 762 & $<0.0001$ & 6.43 & -10 & 48 & 6 & Inferior frontal gyrus (ipsilateral) & 337 & $<0.0001$ & 5.98 & 52 & 10 & 34 \\
\hline Amygdala (right) & 88 & 0.006 & 6.29 & 28 & 4 & -20 & Brainstem & 90 & 0.005 & 5.57 & -8 & -36 & -32 \\
\hline Cerebellar vermis (left) & 85 & 0.006 & 6.19 & -2 & -58 & -34 & Posterior thalamus (ipsilateral) & 111 & 0.003 & 5.48 & 12 & -20 & 16 \\
\hline Premotor cortex (left) & 93 & 0.005 & 5.76 & -54 & 4 & 40 & Frontal eye fields (ipsilateral) & 67 & 0.017 & 5.43 & 30 & -2 & 46 \\
\hline Amygdala-hippocampus-midbrain (right) & 142 & 0.001 & 5.75 & 18 & -8 & -10 & Midbrain (contralateral) & 103 & 0.003 & 5.24 & -4 & -30 & -4 \\
\hline Postcentral gyrus (right) & 124 & 0.001 & 5.60 & 50 & -26 & 42 & Amygdala-midbrain-thalamus (ipsilateral) & 108 & 0.003 & 5.22 & 14 & -24 & -14 \\
\hline Precuneus (bilateral) & 217 & $<0.0001$ & 5.56 & 2 & -60 & 40 & Middle and inferior frontal gyrus (contralateral) & 176 & $<0.0001$ & 4.92 & -34 & 34 & 34 \\
\hline Inferior frontal gyrus (right) & 320 & $<0.0001$ & 5.30 & 38 & 6 & 30 & Orbitofrontal cortex (ipsilateral) & 57 & 0.028 & 4.80 & 32 & 30 & -18 \\
\hline Midbrain (bilateral) & 96 & 0.005 & 5.15 & -6 & -34 & -26 & & & & & & & \\
\hline Temporal pole (left) & 73 & 0.011 & 4.78 & -40 & 14 & -24 & & & & & & & \\
\hline Middle frontal gyrus (left) & 59 & 0.021 & 4.54 & -38 & 38 & 6 & & & & & & & \\
\hline
\end{tabular}

For patient contrasts, laterality is indicated in reference to lesion site.

Table 6. Paired $t$ test comparing percentage signal change in three spherical ROIs in visual cortex show no significant effects in regions ipsilateral relative to contralateral of amygdala lesion side

\begin{tabular}{|c|c|c|c|c|c|c|c|c|c|}
\hline \multicolumn{7}{|l|}{ Aversive $>$ neutral } & \multicolumn{3}{|l|}{ Erotic $>$ neutral } \\
\hline \multirow[b]{2}{*}{ Brain regions } & \multicolumn{4}{|c|}{ Coordinates } & \multirow{2}{*}{$\begin{array}{l}\text { Mean (SD) } \\
\text { ipsilateral \% signal } \\
\text { change }\end{array}$} & \multirow[b]{2}{*}{$p$ value } & \multirow{2}{*}{$\begin{array}{l}\text { Mean (SD) } \\
\text { contralateral \% signal } \\
\text { change }\end{array}$} & \multirow{2}{*}{$\begin{array}{l}\text { Mean (SD) } \\
\text { ipsilateral \% signal } \\
\text { change }\end{array}$} & \multirow[b]{2}{*}{$p$ value } \\
\hline & $x$ & $y$ & $Z$ & & & & & & \\
\hline Fusiform gyrus & 34 & -34 & -22 & $0.06(0.21)$ & $0.03(0.14)$ & 0.355 & $0.10(0.21)$ & $0.04(0.18)$ & 0.064 \\
\hline Visual association cortex & 30 & -83 & -2 & $0.40(0.48)$ & $0.40(0.44)$ & 0.921 & $0.28(0.37)$ & $0.26(0.42)$ & 0.789 \\
\hline Primary visual cortex & 4 & -80 & -4 & $0.55(0.55)$ & $0.50(0.46)$ & 0.501 & $0.37(0.41)$ & $0.34(0.43)$ & 0.160 \\
\hline
\end{tabular}
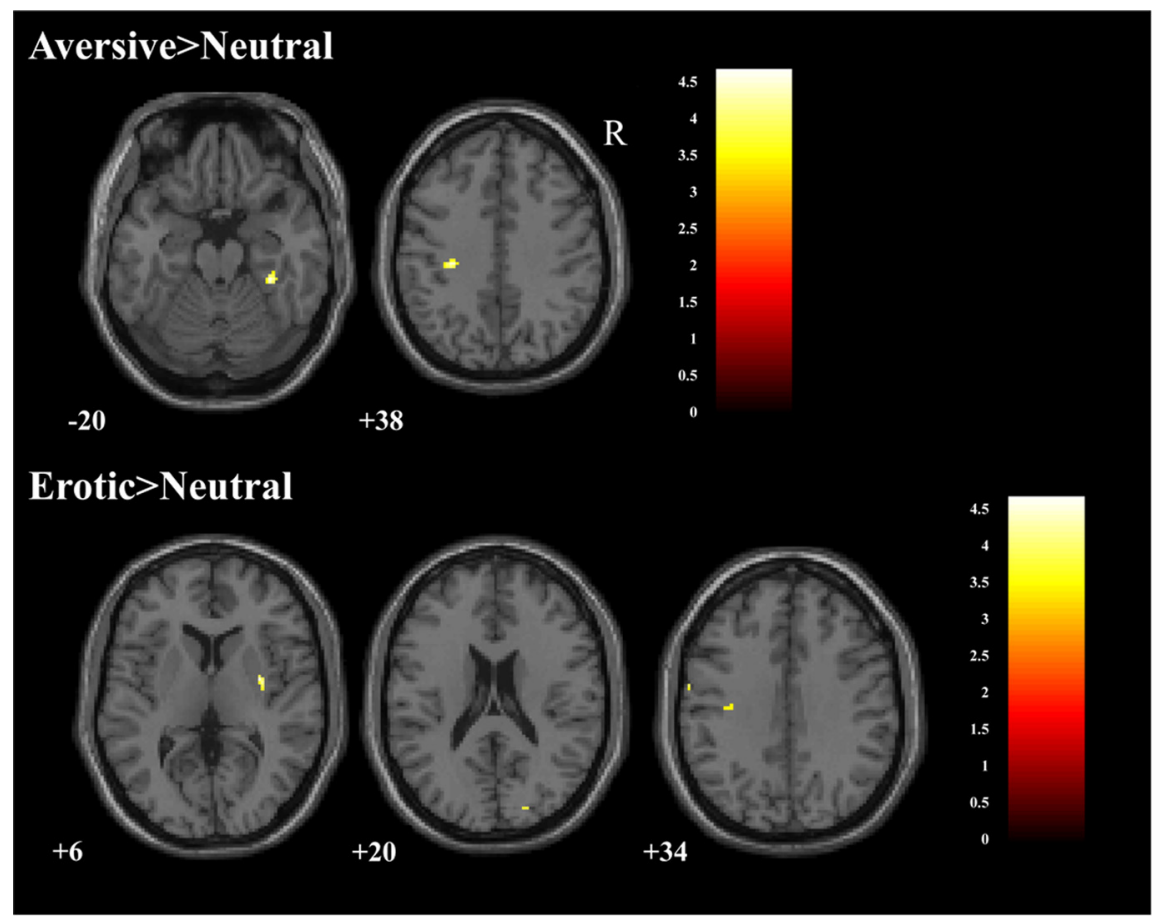

Figure 4. At a reduced statistical threshold $(p<0.001$, cluster $=10)$ areas of increased BOLD signal for control participants relative to the combined patient group for the aversive $>$ neutral (top) and erotic $>$ neutral contrasts (bottom). Color bars represent $t$ values, MNI $y$-plane coordinates in millimeters. associated with stimulus perception, whereas the second peak is associated with downstream modulation based on emotional salience. Discrimination of different waves of visual cortex modulation could prove critical in understanding amygdalar and non-amygdalar sources of modulation. Future studies of patients with amygdala damage using techniques with high temporal resolution, such as magnetoencephalography or electrophysiology will be important in determining whether there are specific temporal features of emotional modulation of sensory cortex that fall under amygdalar control, while other components (such as perhaps sustained modulation) may prove to be independent of the amygdala.

A potential concern in interpreting the results of studies using patients with unilateral damage is whether the amygdala in the nonresected hemisphere might be able to produce a bilateral cortical modulation. While we cannot rule this out, this possibility seems relatively unlikely. Anatomical tracing studies of amygdalar projections to visual areas have focused exclusively on ipsilateral projections (Amaral et al., 2003), and to the extent 


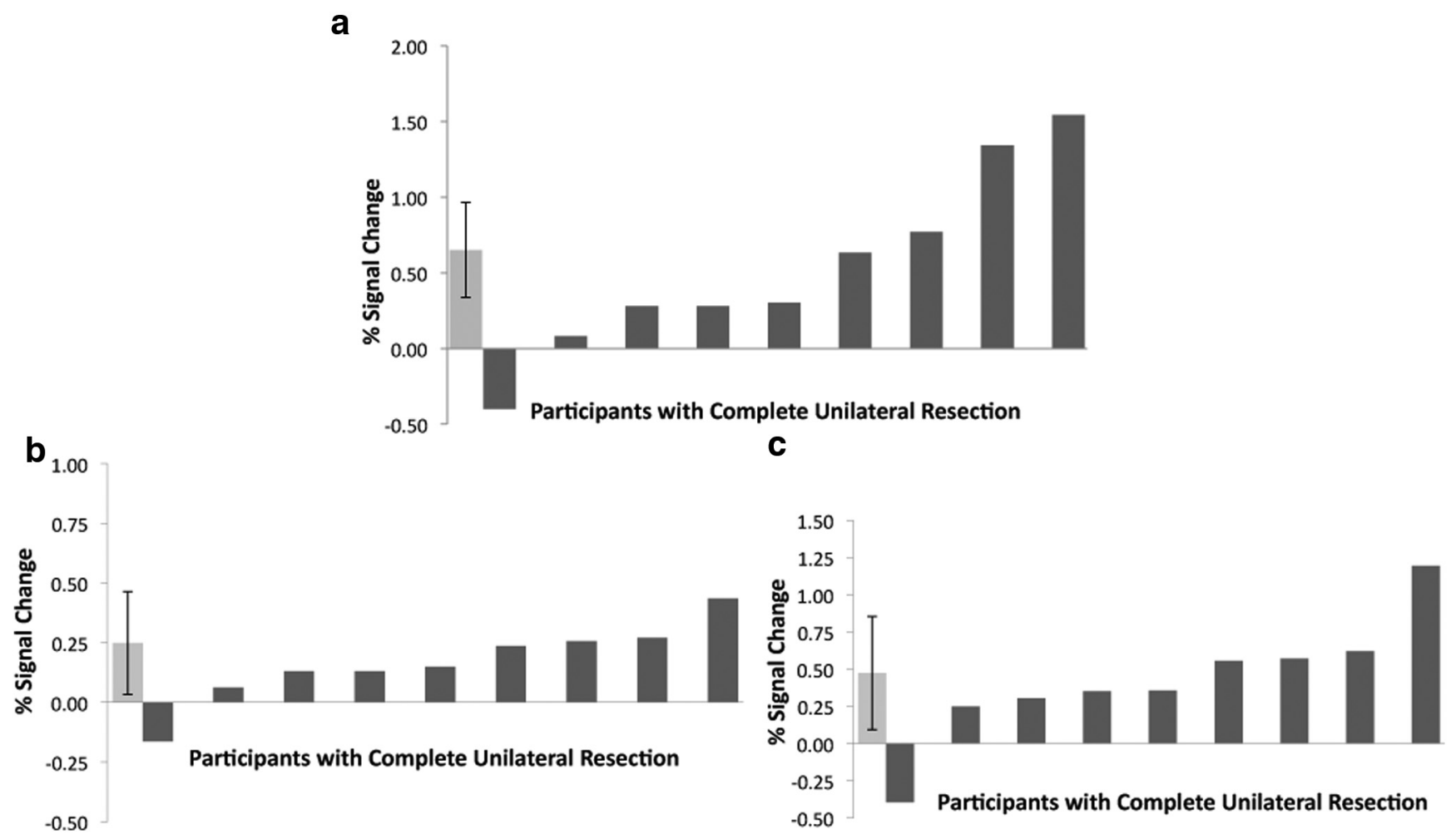

Figure 5. Percentage BOLD signal change during the aversive relative to neutral condition in the (a) primary visual cortex (10 mm diameter spherical ROl at MNI coordinates $4,-80,-4),(\boldsymbol{b})$ ipsilateral fusiform gyrus ( $10 \mathrm{~mm}$ diameter spherical ROI at MNI coordinates $34,-34,-22)$, and (c) contralateral visual association cortex (10 mm diameter spherical ROI at MNI coordinates -30 , $-83,-11)$ in the nine resection patients with complete unilateral amygdala resections. Light gray bar represents mean percentage signal change in control sample. Error bar indicates SD.

Table 7. Peak voxel statistics for regions showing significant positive and negative correlations between percent BOLD signal change and amygdala lesion extent. Laterality is indicated in reference to lesion site

\begin{tabular}{|c|c|c|c|c|c|}
\hline \multirow[b]{2}{*}{ Brain regions (hemisphere)* } & \multirow{2}{*}{$\begin{array}{l}\text { Cluster } \\
\text { size }\end{array}$} & \multicolumn{4}{|c|}{ Peak voxel } \\
\hline & & tscore & $x$ & $y$ & $z$ \\
\hline \multicolumn{6}{|l|}{ Aversive $>$ neutral } \\
\hline \multicolumn{6}{|l|}{ Negative correlations } \\
\hline Posterior lateral thalamus (ipsilateral) & 40 & 6.98 & 20 & -22 & 0 \\
\hline \multicolumn{6}{|l|}{ Positive correlations } \\
\hline Inferior frontal gyrus (ipsilateral) & 222 & -14.38 & 28 & 56 & -2 \\
\hline Precuneus (ipsilateral) & 182 & -10.2 & 4 & -44 & 42 \\
\hline Superior frontal gyrus (ipsilateral) & 80 & -8.62 & 10 & 44 & 36 \\
\hline Fusiform gyrus (contralateral) & 53 & -7.77 & -26 & -40 & -16 \\
\hline Superior parietal cortex (ipsilateral) & 64 & -7.72 & 50 & -44 & 20 \\
\hline Cerebellum (contralateral) & 122 & -7.5 & -16 & -72 & -28 \\
\hline Inferior frontal gyrus (contralateral) & 86 & -6.51 & -26 & 52 & 0 \\
\hline Temporal parietal junction (ipsilateral) & 40 & -5.76 & 38 & -70 & 20 \\
\hline \multicolumn{6}{|l|}{ Erotic $>$ neutral } \\
\hline \multicolumn{6}{|l|}{ Negative correlations } \\
\hline Temporal parietal junction (contralateral) & 45 & 6.61 & -54 & -48 & -6 \\
\hline \multicolumn{6}{|l|}{ Positive correlations } \\
\hline Inferior parietal cortex (contralateral) & 53 & -7.56 & -38 & -82 & 10 \\
\hline Visual association cortex (contralateral) & 60 & -6.59 & -30 & -84 & -10 \\
\hline
\end{tabular}

*For brevity, submaxima are not reported but are available upon request.

that contralateral projections exist, they would be predicted to be relatively weak. Because of this, studies of patients with amygdala sclerosis or resections have particularly focused on contrasts of ipsilateral versus contralateral activity (Benuzzi et al., 2004; Vuilleumier et al., 2004). When we directly contrasted activations in ROIs ipsilateral and contralateral to the lesion, only the fusiform gyrus showed a trend toward lower activation ipsilateral to the lesion, and this was only for the erotic-neutral contrast. Thus, to the extent that lateralized findings emerged, they were highly selective, with primary visual and lateral occipital regions showing no evidence of a decrement in ipsilateral activation.

In theory, one might speculate that the amygdala contralateral to the lesion might drive compensatory responses in its own visual cortex that in turn could modulate responses in the visual cortex of the lesioned hemisphere through interhemispheric projections between the two visual cortices. Although we did not see evidence of any compensatory enhancements in group analyses, regression analyses revealed that greater amygdala damage was related to increased activity in several regions contralateral to the lesion. Of particular note, this pattern was seen in the contralateral fusiform gyrus in the aversive-neutral contrast, and in lateral visual association cortex in the erotic contrast. However, the spatial extent of visual areas showing contralateral enhancement that correlated with lesion extent in the regression analyses was relatively constrained and thus it seems unlikely that a compensatory response driven by the intact amygdala and mediated by interhemispheric connections in the visual cortex can explain the broad spatial distribution of ipsilateral activations in patients.

Because our subjects did not all have complete resections of the amygdala, it might be plausible that the remaining amygdala in the resected hemisphere was enough to produce emotional modulations of the ipsilateral visual system. However, three factors strongly argue against this possibility. First, patients in our study with complete unilateral amygdala loss displayed robust activation of primary and secondary visual areas in response to aversive and erotic images. Second, in regression analyses of the aversive-neutral contrast (in which extent of amygdala resection was entered as a covariate of interest), the posterior thalamus, which may be consistent with the pulvinar and ventral posterior lateral nuclei, was the only ipsilateral region that showed a decline 
in activation that was correlated with the extent of lesion. Indeed, the majority of correlations with lesion extent were in the opposite direction, with multiple ipsilateral and contralateral regions showing greater activations in patients with larger lesions. Interestingly and perhaps in line with our correlational findings, Terburg et al. (2012) reported that partial amygdala lesions can produce heightened processing of emotional stimuli. Finally, the nature of the lesions is such that even though some amygdala nuclei may be intact, the input and output pathways are likely to be severely compromised, as many fibers of passage in the amygdala and in the white matter lateral to the amygdala would be destroyed even if the nuclei are intact.

Our study suggests that intact amygdalae are not necessary for enhanced visual cortex BOLD during viewing of emotional stimuli. However, it is unclear what brain region or regions mediate heightened signal in visual cortex. Others have proposed a possible role for the prefrontal cortex (Pessoa and Adolphs, 2010) or the pulvinar nucleus (Morris et al., 1997) in mediating enhanced visual cortex BOLD responses. Future high temporal resolution functional connectivity studies that examine potential causal pathways outside of the amygdala are needed to clarify the brain networks that mediate enhanced visual cortex BOLD signal in response to emotional stimuli.

\section{References}

Amaral DG, Behniea H, Kelly JL (2003) Topographic organization of projections from the amygdala to the visual cortex in the macaque monkey. Neuroscience 118:1099-1120. CrossRef Medline

Anderson AK, Phelps EA (2001) Lesions of the human amygdala impair enhanced perception of emotionally salient events. Nature 411:305-309. CrossRef Medline

Ashburner J, Friston KJ (2005) Unified segmentation. Neuroimage 26:839851. CrossRef Medline

Bach DR, Talmi D, Hurlemann R, Patin A, Dolan RJ (2011) Automatic relevance detection in the absence of a functional amygdala. Neuropsychologia 49:1302-1305. CrossRef Medline

Benuzzi F, Meletti S, Zamboni G, Calandra-Buonaura G, Serafini M, Lui F, Baraldi P, Rubboli G, Tassinari CA, Nichelli P (2004) Impaired fear processing in right mesial temporal sclerosis: a fMRI study. Brain Res Bull 63:269-281. CrossRef Medline

Britton JC, Taylor SF, Sudheimer KD, Liberzon I (2006) Facial expressions and complex IAPS pictures: common and differential networks. Neuroimage 31:906-919. CrossRef Medline

Chumbley JR, Friston KJ (2009) False discovery rate revisited: FDR and topological inference using Gaussian random fields. Neuroimage 44:6270. CrossRef Medline

Crinion J, Ashburner J, Leff A, Brett M, Price C, Friston K (2007) Spatial normalization of lesioned brains: performance evaluation and impact on fMRI analyses. Neuroimage 37:866-875. CrossRef Medline

Das P, Kemp AH, Liddell BJ, Brown KJ, Olivieri G, Peduto A, Gordon E, Williams LM (2005) Pathways for fear perception: modulation of amygdala activity by thalamo-cortical systems. Neuroimage 26:141-148. CrossRef Medline

Fairhall SL, Ishai A (2007) Effective connectivity within the distributed cortical network of face perception. Cereb Cortex 17:2400-2406. Medline

Fitzgerald DA, Angstadt M, Jelsone LM, Nathan PJ, Phan KL (2006) Beyond threat: amygdala reactivity across multiple expressions of facial affect. Neuroimage 30:1441-1448. CrossRef Medline

Hadj-Bouziane F, Liu N, Bell AH, Gothard KM, Luh WM, Tootell RB, Murray EA, Ungerleider LG (2012) Amygdala lesions disrupt modulation of functional MRI activity evoked by facial expression in the monkey inferior temporal cortex. Proc Natl Acad Sci U S A 109:E3640-36408. CrossRef Medline

Honeycutt NA, Smith PD, Aylward E, Li Q, Chan M, Barta PE, Pearlson GD (1998) Mesial temporal lobe measurements on magnetic resonance imaging scans. Psychiatry Res 83:85-94. CrossRef Medline

Krolak-Salmon P, Hénaff MA, Vighetto A, Bertrand O, Mauguière F (2004) Early amygdala reaction to fear spreading in occipital, temporal, and fron- tal cortex: a depth electrode ERP study in human. Neuron 42:665-676. CrossRef Medline

Lang PJ, Bradley MM, Fitzsimmons JR, Cuthbert BN, Scott JD, Moulder B, Nangia V (1998) Emotional arousal and activation of the visual cortex: an fMRI analysis. Psychophysiology 35:199-210. CrossRef Medline

Lang PJ, Bradley MM, Cuthbert BN (2008) International affective picture system (IAPS): affective ratings of pictures and instruction manual. Gainesville, FL: University of Florida. Technical Report A-8.

Morris JS, Friston KJ, Dolan RJ (1997) Neural responses to salient visual stimuli. Proc Biol Sci 264:769-775. CrossRef Medline

Morris JS, Ohman A, Dolan RJ (1998) Conscious and unconscious emotional learning in the human amygdala. Nature 393:467-470. CrossRef Medline

Peck CJ, Lau B, Salzman CD (2013) The primate amygdala combines information about space and value. Nat Neurosci 16:340-348. CrossRef Medline

Pessoa L, Adolphs R (2010) Emotion processing and the amygdala: from a 'low road' to 'many roads' of evaluating biological significance. Nat Rev Neurosci 11:773-783. CrossRef Medline

Phan KL, Taylor SF, Welsh RC, Ho SH, Britton JC, Liberzon I (2004) Neural correlates of individual ratings of emotional salience: a trial-related fMRI study. Neuroimage 21:768-780. CrossRef Medline

Phelps EA, Ling S, Carrasco M (2006) Emotion facilitates perception and potentiates the perceptual benefits of attention. Psychol Sci 17:292-299. CrossRef Medline

Piech RM, Lewis J, Parkinson CH, Owen AM, Roberts AC, Downing PE, Parkinson JA (2010) Neural correlates of affective influence on choice. Brain Cogn 72:282-288. CrossRef Medline

Piech RM, McHugo M, Smith SD, Dukic MS, Van Der Meer J, Abou-Khalil B, Most SB, Zald DH (2011) Attentional capture by emotional stimuli is preserved in patients with amygdala lesions. Neuropsychologia 49:33143319. CrossRef Medline

Pruessner JC, Li LM, Serles W, Pruessner M, Collins DL, Kabani N, Lupien S, Evans AC (2000) Volumetry of hippocampus and amygdala with highresolution MRI and three-dimensional analysis software: minimizing the discrepancies between laboratories. Cereb Cortex 10:433-442. CrossRef Medline

Reinders AA, den Boer JA, Büchel C (2005) The robustness of perception. Eur J Neurosci 22:524-530. CrossRef Medline

Sabatinelli D, Lang PJ, Bradley MM, Costa VD, Keil A (2009) The timing of emotional discrimination in human amygdala and ventral visual cortex. J Neurosci 29:14864-14868. CrossRef Medline

Sabatinelli D, Fortune EE, Li Q, Siddiqui A, Krafft C, Oliver WT, Beck S, Jeffries J (2011) Emotional perception: meta-analyses of face and natural scene processing. Neuroimage 54:2524-2533. CrossRef Medline

Tamietto M, de Gelder B (2010) Neural bases of the non-conscious perception of emotional signals. Nat Rev Neurosci 11:697-709. CrossRef Medline

Terburg D, Morgan BE, Montoya ER, Hooge IT, Thornton HB, Hariri AR, Panksepp J, Stein DJ, van Honk J (2012) Hypervigilance for fear after basolateral amygdala damage in humans. Transl Psychiatry 2:e115. CrossRef Medline

Todd RM, Talmi D, Schmitz TW, Susskind J, Anderson AK (2012) Psychophysical and neural evidence for emotion-enhanced perceptual vividness. J Neurosci 32:11201-11212. CrossRef Medline

Tsuchiya N, Moradi F, Felsen C, Yamazaki M, Adolphs R (2009) Intact rapid detection of fear faces in the absence of the amygdala. Nat Neurosci 12:1224-1225. CrossRef Medline

Vuilleumier P, Richardson MP, Armony JL, Driver J, Dolan RJ (2004) Distant influences of amygdala lesion on visual cortical activation during emotional face processing. Nat Neurosci 7:1271-1278. CrossRef Medline

Wager TD, Keller MC, Lacey SC, Jonides J (2005) Increased sensitivity in neuroimaging analyses using robust regression. Neuroimage 26:99-113. CrossRef Medline

Wendt J, Weike AI, Lotze M, Hamm AO (2011) The functional connectivity between amygdala and extrastriate visual cortex activity during emotional picture processing depends on stimulus novelty. Biol Psychol 86:203-209. CrossRef Medline

Yiend J (2010) The effects of emotion on attention: a review of attentional processing of emotional information. Cogn Emotion 24:3-47. CrossRef

Zald DH (2003) The human amygdala and the emotional evaluation of sensory stimuli. Brain Res Brain Res Rev 41:88-123. CrossRef Medline 